\title{
Wear of Fluorapatite Single Crystals: IV. Influence of Sliding Direction on Frictional Behavior and Surface Failure
}

\author{
J. M. POWERS, K. C. LUDEMA, and R. G. CRAIG \\ School of Dentistry and College of Engineering, University of \\ Michigan, Ann Arbor, Michigan 48104, USA
}

Frictional anisotropy of the basal plane of natural fluorapatite single crystals was observed; the coefficient of friction $(\beta)$ in the [2110] direction $(\beta=0.217)$ was approximately $12 \%$ higher than that in the $[01 \overline{1} 0]$ direction $(\beta=0.193)$. The load at which the ductile-to-brittle transition occurred $(\Omega)$ was significantly higher in the $[2 \overline{1} \overline{1} 0]$ direction $(\Omega=77 \mathrm{gm})$ than in the $[01 \overline{1} 0]$ direction $(\Omega=15 \mathrm{gm})$. A mechanism is proposed involving the maximum normal stress and probable cleavage planes.

Preliminary studies of the wear of fluorapatite single crystals subjected to sliding neglected the possible influence of crystallographic direction on the frictional behavior and surface failure of the basal plane. ${ }^{1-3}$ In these studies, possible anisotropic effects were considered to contribute to the experimental error in the analysis of the data. Buckley 4 has observed that friction coefficients (with limited plowing components) for a number of metals in sliding contact with other metals were lowest on the preferred slip or glide plane in the preferred slip direction. The anisotropy of surface failure has been discussed to a limited extent by Steijn ${ }^{5}$ for sapphire and by Bowden. Brookes, and Hanwell 6 and Bowden and Brookes $^{7}$ for magnesium oxide single crystals.

The purpose of this study was to evaluate

Based on a dissertation submitted in partial fulfillment of the requirements for the PhD degree in the Horace H. Rackham School of Graduate Studies at the Unicersity of Michigan, 1972.

This paper was presented, in part, at the 50th gen eral session of the IADR in Las Vegas, Nevada, March 1972.

This investigation was supported, in part, by USPHS Training Grant DE.00181 from the National Institute of Dental Research, National Instituces of Health. Bethesda, Md.

Received for publication May 10, 1972. the influence of sliding direction on the frictional behavior and surface failure of the basal plane of fluorapatite single crystals.

\section{Materials and Methods}

A diamond hemisphere (360 micrometers $[\mu \mathrm{m}]$ in diameter) ${ }^{a}$ was slid across the basal surface of natural fluorapatite (FAP) single crystals." Fifteen parallel, one-traversal scratches resulted from sliding a normal load of 10 to $150 \mathrm{gm}$ in increments of 10 $\mathrm{gm}$. The scratches were made on the basal plane of each of six crystals (FAP, 1 to 6) in the $x$ direction $[2 \overrightarrow{110}$ and subsequently in the $y$ direction $[01 \overline{1} 0]$. Before sliding in the $y$ direction, the crystals were repolished. The $x$ direction was chosen as that direction on the basal plane parallel to what was considered to be a prismatic [10̄0] surface of the natural crystal. The $y$ direction then was chosen as the direction perpendicular to this $[10 \overline{1} 0]$ surface. All runs were made in air (absorbed water). The single crystals were given a polishing and surface treatment before use. ${ }^{1}$ The apparatus used for scratching the surface of a specimen and measuring the tangential force has been described in detail.1.2 The failure classification scale used in this study also has been reported. ${ }^{3}$

Tangential force data were collected during each run. The track width and failure classification of each scar were determined under optical magnification on a metallograph.c To analyze the tangential force vs normal load data. a linear regression model was chosen. 8 We assumed that the tangential force vs normal load curve was a straight line that passed through the origin.

\footnotetext{
" Wheel Trueing Tool Co., Detroit, Mich.

b Southwest Scientific Co., Hamilton, Mont.

c Aristophot, Ernst Ieitz, Wetzlar. W Ger.
} 
Analysis of variance was used to analyze the data further. From the failure classification data, the maximum normal load above which a ductile mode of failure (Class 1) was no longer observed $(\Omega)$ was chosen.

\section{Results}

Data for failure classification, track width, and tangential force are plotted as functions of a normal load in Figures 1 and 2 for sliding on FAPl in the $x$ and $y$ directions, respectively. Several curves calculated from models were compared with the measured data on these plots. A linear regression line was fit to the tangential force vs normal load data. The slope $(\beta)$ of this regression line and its standard error are indicated on the plot. The measured values of track width were compared qualitatively with values computed from an equation derived from a special case of Hertz's theory of contact between two elastic spheres. ${ }^{d}$ In addition to $\beta$ and $\Omega$, several other parameters are reported in Tables 1 and 2 for sliding in the $x$ and $y$ directions on the set of six crystals. Values of $W$ and $F$, the tangential force, were evaluated at $W=\Omega$. From the value of $W(\Omega)$ and the geometry of the slider, the track depth evaluated at $\Omega, d(\Omega)$, was calculated. The following parameterse derived from Hertzian theory also were evaluated at $W=\Omega: z$, the distance below the surface of the crystal at which shear stress is a maximum; $p^{\prime}$, the maximum normal pressure; $\tau_{\max }$, the maximum shear stress; $\tau_{\text {houndary }}$, the shear stress at the boundary of

\footnotetext{
d The equation' used was:

$w=1.816(W \mathrm{R})^{1 / 3}\left[\frac{E_{B}\left(1-\nu_{A}{ }^{2}\right)+E_{A}\left(1-\nu_{B}\right)}{E_{A} E_{B}}\right]^{1 / 3}$,
}

where $w$ equals track width, $W$ equals normal load, $R$ equals the radius of the diamond hemisphere, and $\nu$ and $E$ are the Poisson's ratio and Young's modulus of fluorapatite $(A)$ and diamond $(B)$, respectively. In this equation Poisson's ratio and Young's modulus for diamond were 0.30 and $9.48 \times 10^{4} \mathrm{~kg} / \mathrm{mm}^{2}$, respectively. Poisson's ratio for fluorapatite was 0.28. Two values of Young's modulus were used to indicate a range of values for fluorapatite. A value of $8.44 \times 10^{3}$ $\mathrm{kg} / \mathrm{mm}^{2}$ was chosen for comparative purposes as representative of an average value for human enamel. The value of $1.47 \times 10^{4} \mathrm{~kg} / \mathrm{mm}^{2}$ represented Young's modulus in the [0001] direction for a single crystal of Huorapatite. ${ }^{10}$

- These parameters, with the exception of $\sigma \mu$, have been discussed in detail by Hubert1 and Timoshenko and Goodier. ${ }^{12}$ The effect of an applied tangential force on the stress distribution at the contact area between two elastic bodies loaded normally has been discussed by Mindlin. ${ }^{33}$

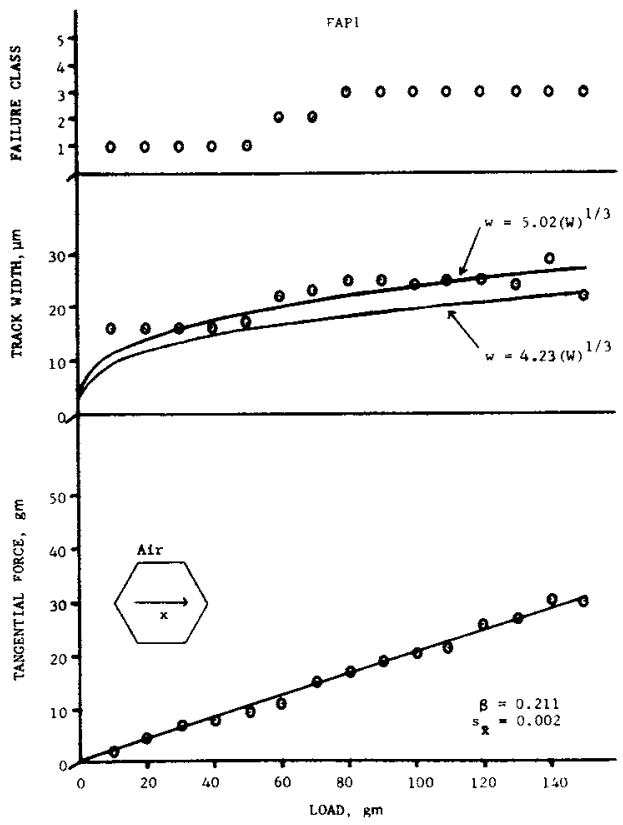

FIG 1,-Failure classification, track width, and tangential force vs normal load for FAPI in air in $x$ sliding direction.

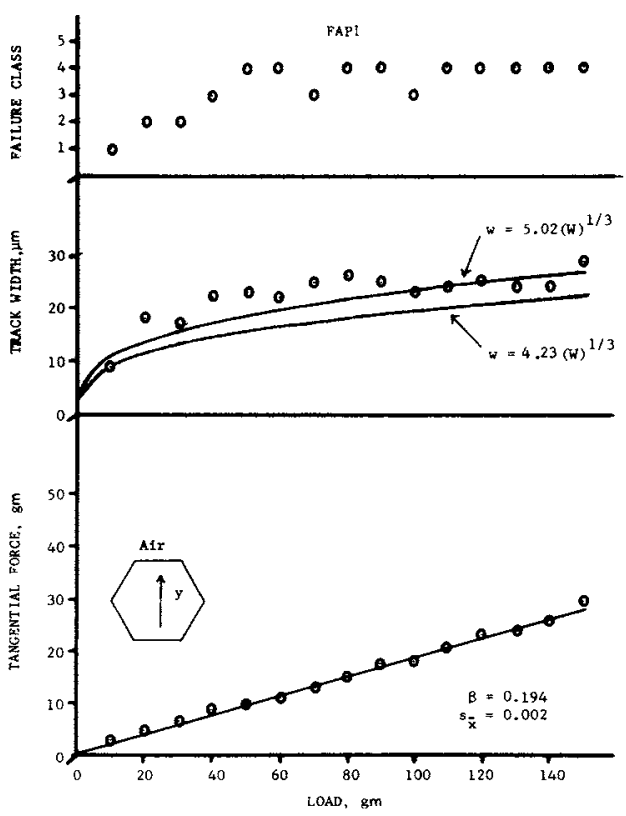

Fig 2.-Failure classification, track width, and tangential force vs normal load for FAPI in air in $y$ sliding direction. 
TABLE 1

Properties of FAP (1 to 6) at the Ductile-to-Brittle Transition as a Function of Sliding Direction

\begin{tabular}{lcccrrrr}
\hline \hline Crystal & $\begin{array}{c}\text { Sliding } \\
\text { Direction }\end{array}$ & $\beta$ & $\begin{array}{c}\Omega \\
(\mathrm{gm})\end{array}$ & $\begin{array}{c}F(\Omega) \\
(\mathrm{gm})\end{array}$ & $\begin{array}{c}w(\Omega) * \\
(\mu \mathrm{m})\end{array}$ & $\begin{array}{c}z(\Omega) \dagger \\
(\mu \mathrm{m})\end{array}$ & $\begin{array}{c}d(\Omega) \ddagger \\
(\mu \mathrm{m})\end{array}$ \\
\hline FAP1 & $\boldsymbol{x}$ & 0.211 & 50 & 10.5 & 15.6 & 3.7 & 0.17 \\
& $y$ & 0.194 & 10 & 1.9 & 9.1 & 2.2 & 0.06 \\
FAP2 & $\boldsymbol{y}$ & 0.187 & 60 & 11.2 & 16.6 & 4.0 & 0.19 \\
& $y$ & 0.180 & 20 & 3.6 & 11.5 & 2.8 & 0.09 \\
FAP3 & $x$ & 0.216 & 10 & 2.2 & 9.1 & 2.2 & 0.06 \\
& $y$ & 0.192 & 10 & 1.9 & 9.1 & 2.2 & 0.06 \\
FAP4 & $\boldsymbol{x}$ & 0.245 & 120 & 29.4 & 20.8 & 5.0 & 0.30 \\
& $y$ & 0.188 & 10 & 1.9 & 9.1 & 2.2 & 0.06 \\
FAP5 & $x$ & 0.237 & $\geqslant 150$ & $\geqslant 35.6$ & $\geqslant 22.5$ & $\geqslant 5.4$ & $\geqslant 0.36$ \\
& $y$ & 0.193 & 30 & 5.8 & 13.1 & 3.2 & 0.12 \\
FAP6 & $\boldsymbol{x}$ & 0.208 & 70 & 14.6 & 17.4 & 4.2 & 0.21 \\
& $y$ & 0.213 & $<10$ & $<2.1$ & $<9.1$ & $<2.2$ & $<0.06$ \\
\hline
\end{tabular}

- Track width calculated from slider geometry and Hertzian model at $W=\Omega$.

$\dagger z$ at $\tau_{\max }$ for Hertzian behavior at $W=\Omega$.

$\ddagger$ Track depth calculated from Hertzian track widths and slider geometry at $W=\Omega$.

the circle of contact; and $\sigma_{\mu}$, the tangential traction stress at the center of the surface of contact evaluated at $\mu=\beta$, where $\mu$ is a constant coefficient of friction.

An analysis of variance was used to determine if there was a difference among values of $\beta$ indicated in Table 2. The analysis of variance was based on a twofactor design; the factors of specimens and sliding directions were studied at six and two levels, respectively. The main effect of specimens was found to be significant, since $F=45.4>F_{(5 . \infty)}=4.10$, at the 0.001 level. It was deduced that there were differences among the six crystals. The main effect of sliding direction was significant, since $F=$ $268>F_{(1 . x)}=10.8$, at the 0.001 level. There was a difference between the two levels of sliding direction. The interaction between crystals and sliding direction, how-

TABLE 2

Stress Properties of FAP ( 1 to 6) at the Ductile-to-Brittle Transition as a Function of Sliding, Direction

\begin{tabular}{|c|c|c|c|c|c|c|}
\hline Crystal & $\begin{array}{c}\text { Sliding } \\
\text { Direction }\end{array}$ & $\underset{(\mathrm{gm})}{\Omega}$ & $\begin{array}{c}p^{\prime}(\Omega)^{*} \\
\left(\mathrm{~kg} / \mathrm{mm}^{2}\right)\end{array}$ & $\begin{array}{l}\tau \max (\Omega) \dagger \\
\left(\mathrm{kg} / \mathrm{mm}^{2}\right)\end{array}$ & $\begin{array}{c}\tau_{\text {boundary }}(\Omega) \ddagger \\
\left(\mathrm{kg} / \mathrm{mm}^{2}\right)\end{array}$ & $\begin{array}{c}\sigma \mu(\Omega) \S \\
\left(\mathrm{kg} / \mathrm{mm}^{2}\right)\end{array}$ \\
\hline \multirow[t]{2}{*}{ FAPl } & $x$ & 50 & 393 & 124.0 & 57.6 & 82.9 \\
\hline & $y$ & 10 & 230 & 72.4 & 33.7 & 44.6 \\
\hline \multirow[t]{2}{*}{ FAP2 } & $x$ & 60 & 418 & 132.0 & 61.2 & 78.2 \\
\hline & $y$ & 20 & 290 & 91.2 & 42.5 & 52.2 \\
\hline \multirow[t]{2}{*}{ FAP3 } & $x$ & 10 & 230 & 72.4 & 33.7 & 49.7 \\
\hline & $y$ & 10 & 230 & 72.4 & 33.7 & 44.2 \\
\hline \multirow[t]{2}{*}{ FAP4 } & $x$ & 120 & 526 & 166.0 & 77.2 & 129.0 \\
\hline & $y$ & 10 & 230 & 72.4 & 33.7 & 43.2 \\
\hline \multirow[t]{2}{*}{ FAP5 } & $x$ & $\geqslant 150$ & $\geqslant 567$ & $\geqslant 178.0$ & $\geqslant 83.1$ & $\geqslant 134.0$ \\
\hline & $y$ & 30 & 331 & 104.0 & 48.6 & 63.9 \\
\hline \multirow[t]{2}{*}{ FAP6 } & $x$ & 70 & 440 & 138.0 & 64.5 & 91.5 \\
\hline & $y$ & $>10$ & $>230$ & $>72.4$ & $>33.7$ & $>49.0$ \\
\hline
\end{tabular}

- Maximum normal pressure at $W=\Omega$ calculated from Hertzian model.

+ Maximum shearing stress (at $z$ ) at $W=\Omega$ calculated from Hertzian model.

I Shearing stress at boundary of circle of contact at $W=\Omega$ calculated from Hertzian model.

$\$$ Tangential traction at $W=\Omega$ with $\mu=\beta$ at the center of the surface of contact. 
ever, also was found to be significant, since $F=41.7>F_{(\bar{j}, \infty)}=4.10$, at the 0.001 level. This interaction meant that the behavior of $\beta$ could be predicted statistically as a function of sliding direction only if the crystals were regarded as a sample of a larger population of crystals. The error associated with the analysis was $1.2 \%$. The confidence interval that could be used to determine the difference between the means of the two sliding directions for a given crystal was 0.007 at the 0.05 level of significance. as determined by the method of Duncan. ${ }^{14}$

Based on the assumption that the six fluorapatite crystals were representative of a sample of a larger population of crystals, a one-way analysis of variance was made for each of the ten parameters listed in Tables 1 and 2. The means and standard errors for these parameters are listed in Table 3 . The factor of sliding direction was tested at two levels, the $x$ and $y$ directions, with six replications per cell. The tangential force coefficient, $\beta$, in the $x$ sliding direction was statistically different $(P<0.05)$ than that in the $y$ sliding direction and approximately $120^{\circ}$ higher in magnitude. The mode of

TABLE 3

Average Behavior of FAP (I to 6) as a Function of SLIDING DiRECTION

\begin{tabular}{|c|c|c|}
\hline Property & $\begin{array}{l}x \text { Sliding } \\
\text { Direction }\end{array}$ & $\begin{array}{l}y \text { Sliding } \\
\text { Direction }\end{array}$ \\
\hline$\beta$ & $0.217(0.008) *$ & $0.193(0.004)$ \\
\hline $\begin{array}{c}\Omega \\
(\mathrm{gm})\end{array}$ & $77(21)$ & $15(3)$ \\
\hline $\begin{array}{c}F(\Omega) \\
(\mathrm{gm})\end{array}$ & $17.2(5.2)$ & $2.9(0.6)$ \\
\hline $\begin{array}{c}w(\Omega) \\
(\mu \mathrm{m})\end{array}$ & $17.0(1.9)$ & $10.2(0.7)$ \\
\hline $\begin{array}{l}z(\Omega) \\
(\mu \mathrm{m})\end{array}$ & $4.1(0.5)$ & $2.5(0.2)$ \\
\hline $\begin{array}{l}d(\Omega) \\
(\mu \mathrm{m})\end{array}$ & $0.21(0.04)$ & $0.07(0.01)$ \\
\hline $\begin{array}{c}p^{\prime}(\Omega) \\
\left(\mathrm{kg} / \mathbf{m m}^{2}\right)\end{array}$ & $429(48)$ & 257 (18) \\
\hline $\begin{array}{c}\tau_{\max }(\Omega) \\
\left(\mathrm{kg} / \mathrm{mm}^{2}\right)\end{array}$ & $135(15)$ & $80.8(5.6)$ \\
\hline $\begin{array}{c}\tau_{\text {boundary }}(\Omega) \\
\left(\mathrm{kg} / \mathrm{mm}^{2}\right)\end{array}$ & $62.9(7.1)$ & $37.6(2.6)$ \\
\hline $\begin{array}{c}\sigma_{\mu}(\Omega) \\
\left(\mathrm{kg} / \mathrm{mm}^{2}\right)\end{array}$ & $94.2(13.1)$ & $49.5(3.2)$ \\
\hline
\end{tabular}

- Standard error of the mean is in parentheses. surface failure remained ductile to a higher load $(\Omega)$ in the $x$ sliding direction. The remainder of the parameters in the $x$ sliding direction were likewise statistically different $(P<0.05)$ than those in the $y$ sliding direc. tion. An average coefficient of variation associated with the analysis was $39 \%$.

It was interesting to note the trend of comparable variables within a specific column (sliding direction) of Table 3. We observed that $z(\Omega)$ was an order of magnitude larger than $d(\Omega)$. The variables describing stress based on the Hertzian model could be ranked in the following order: $p^{\prime}(\Omega)>\tau_{\max }(\Omega)>\sigma_{\mu}(\Omega)>\tau_{\text {lemulary }}(\Omega)$.

\section{Discussion}

Natural fluorapatite has imperfect basal and $\{10 \overline{10} 0\}$ cleavage. ${ }^{15}$ Melt-grown $(0001)$ fluorapatite crystals have been observed to cleave on both basal and $\{1 \overline{2} 10\}$ planes. ${ }^{16}$ The tendency toward cleavage was enhanced by conditions that favored inclusion formation, that is, high growth rates and nonideal composition melts. For melt-grown fluorapatite crystals, McManus, Hopkins, and Takei17 contend that slip takes place on $\{10 \overline{1} 0\}$ prism planes with one Burger's vector in the [0001] direction and another Burger's vector in the basal plane, presumably in the [1010] prism direction. Hopkins ${ }^{18}$ has reported that dislocations where Burger's vectors lie in the basal plane of fluorapatite would have energies about double that of those with a Burger's vector parallel to [0001].

The tangential force coefficient, $\beta$ (Table 3 , was approximately $12 \%$ larger in magnitude for sliding in the $x$ direction, than for sliding in the $y$ direction. ${ }^{f}$ This frictional anisotropy of fluorapatite was neglected in a previous study of the frictional. behavior of fluorapatite. ${ }^{2}$ The degree of anisotropy observed is consistent with the observations made by Bowden and Brookes ${ }^{\bar{\tau}}$ for the frictional behavior of $\mathrm{MgO}$ single crystals. They suggested that a critical amount of subsurface deformation was necessary for aniso-

I On the basis of crystal geometry, the $x$ sliding direction was presumed to be parallel to a $\langle 2 \overline{1} 10\rangle$ direction. The $y$ sliding direction was presumed to be parallel to a $\langle 0 \mid \overrightarrow{10}\rangle$ direction. This assumption was supported by the orientation of etch pits, the hexagonal sides of which were oriented parallel to the prism faces of the crystal. 18 
tropic friction, although for magnesium oxide, strain was accommodated primarily by plastic deformation over the load range studied. Since subsurface fracture was observed even at the lowest loads in the present study, we propose that the criterion of critical subsurface deformation was met under the experimental conditions examined. The lower friction in the $y$ direction is consistent with the hypothesis proposed by Buckley ${ }^{4}$ that lower coefficients of friction can be expected for sliding in preferred slip directions for materials with limited plowing components. In the present study, the $y$ direction was parallel to a slip direction, although the $\{01 \overline{1} 0\}<01 \overline{1} 0>$ slip system is less energetically favorable than the $\{01 \overrightarrow{1} 0\}[0001]$ system. ${ }^{18}$

Statistical analysis of the track width data for the $x$ and $y$ sliding directions was hindered by the scatter of the data at low loads. This scatter apparently was due to roughness of the diamond slider. The roughness resulted in outlying scratches, presumably caused by "bumps" or asperities located beyond the circle of contact defined by the Hertzian analysis." Nevertheless, these outlying scratches were included in the measurement of track width; thus, at low loads, larger values of track width were measured than should have been. The net effect caused the results of regression analysis to be difficult to interpret.

Qualitative interpretation of the track width data suggested that there probably was little difference between the values measured for the $x$ and $y$ sliding directions. The fit of these values to the calculated curves was reasonably good, except for values measured at low loads. These observations are consistent with the fact that elastic properties of hexagonal crystals are symmetrical around the $c$ axis. Any variation in track width that might be observed would necessarily be the result of variation in the degree of plastic deformation attainable in a given direction; however. such variation was not observed.

The value of the maximum normal load above which a ductile mode of surface failure is no longer observed was significantly

In the present study, the contact area between the diamond slider and the basal plane of FAP was assumed to be circular. lower in the $y$ sliding direction, as indicated in Table 3. This observation can be interpreted to mean that the ductile-to-brittle transition occurs at a lower load in the $y$ sliding direction. Consistent with this is the observation that the values of $p^{\prime}(\Omega), \tau_{\max }$ $(\Omega), \sigma_{\mu}(\Omega)$, and $\tau_{\text {boundary }}(\Omega)$ were lower in the $y$ sliding direction. Presumably, the initiation of subsurface failure, which occurred to a greater extent in the $y$ sliding direction, is influenced predominantly by the magnitude by $\tau_{\max }$. On the other hand, the initiation of surface fracture could be influenced by $\tau_{\text {boundary }}$ or by $\sigma_{\mu}$. As indicated in Table 3, $\sigma_{\mu}$ was observed to be greater in magnitude than $\tau_{\text {boundary. }}$ Therefore, the magnitude of $\sigma_{\mu}$ probably controls the initiation of surface failure under the experimental conditions examined.

The anisotropy of surface failure has been discussed only to a limited extent in the literature. The existence of the ductile-tobrittle transition for the basal plane of FAP was reported by Powers and Craig ${ }^{3}$; however, they failed to identify direction of sliding as a variable in that study. The scatter of their fracture classification data may now be explained. Steijn ${ }^{5}$ found that the wear of the basal plane of sapphire was independent of sliding direction, although wear of a prismatic plane resulted in severe chipping in a [0001] direction but gave a smooth wear surface in the $[\overline{2} 110]$ direction. Bowden, Brookes, and Hanwell, ${ }^{6}$ and Bowden and Brookes ${ }^{7}$ observed that the orientation of chevrons and tensile cracks in magnesium oxide single crystals depended on sliding direction. In the present study, surface cracks formed during sliding in the $y$ direction were oriented $30^{\circ}$ from the track and pointed toward the origin of sliding. Cracks formed during sliding in the $x$ direction were oriented at an angle larger than $45^{\circ}$ and pointed toward the origin of sliding.

The following model is proposed to explain the fracture behavior observed for sliding on the basal plane of FAP. This model is diagrammed in Figure 3. For sliding in the $y$ direction (Fig 3,b), the complex stress state is such that imperfect cleavage occurs on $(10 \overline{1} 0)$ and $(1 \overline{1} 00)$ planes, that is, those planes oriented at a $30^{\circ}$ angle to the direction of sliding. For sliding in the $x$ direction (Fig 3,a), the same stress state 

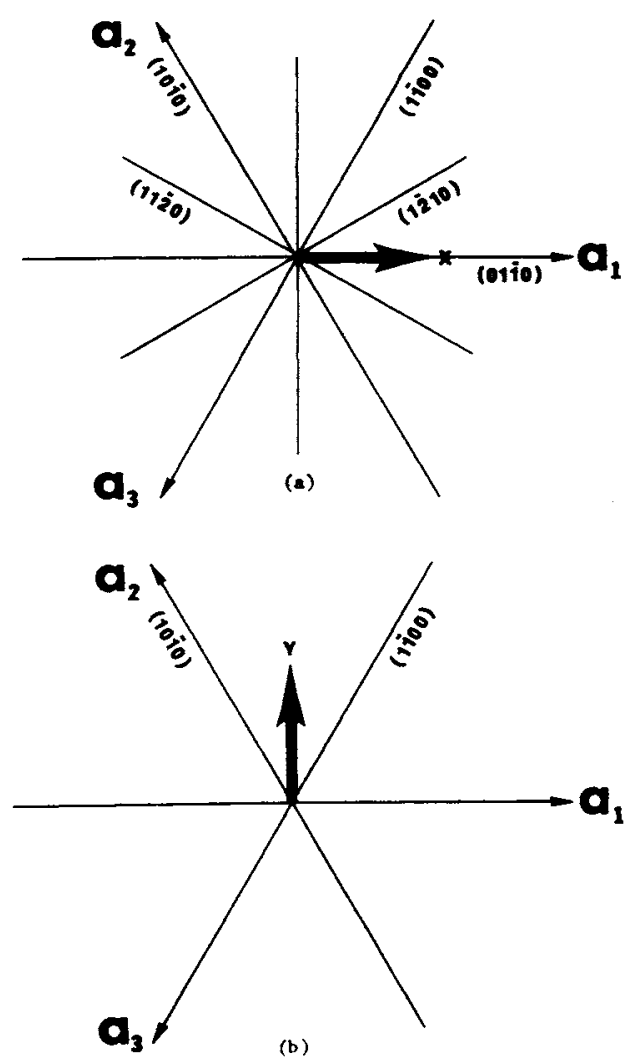

Fic 3.-Diagram of hexagonal system shows orientation of cleavage planes with respect to $x$ sliding direction $(a)$ and $y$ sliding direction (b) .

would favor cleavage on those planes oriented at a $30^{\circ}$ angle to the direction of sliding, that is, the $(1 \overline{2} 10)$ and $(1 \overline{1} \overline{0} 0)$ planes. However, cleavage on $\{\overline{2} 110\}$ planes, although observed for melt-grown crystals, was not observed for natural FAP. Furthermore. cleavage on $\{\overline{2} 110\}$ planes should be less favorable energetically than cleavage on $\{0 \overrightarrow{1} 0\}$ planes because of the larger number of strongly bound phosphate tetrahedra overlapping $\{\overline{2} 110\}$. Thus, the only cleavage planes oriented favorably to accommodate the stress imposed as a result of sliding in the $x$ direction are the $(1 \overline{100})$ and $(10 \overrightarrow{10})$ planes. Because they are oriented at less favorable angle of $60^{\circ}$ to the sliding direction, a larger maximum normal stress would be required to initiate cracking in this $\mathrm{di}$ - rection. Cracks that occurred infrequently at an angle of $30^{\circ}$ for sliding in the $x$ direction were probably the result of $\{\overline{2} 110\}$ cleavage, perhaps on a plane where a natural flaw existed. The fact that most cracks were observed at a $45^{\circ}$ angle to the wear track suggests that for sliding in the $x$ direction, the maximum normal stress is oriented to cause cracking at an angle of $45^{\circ}$ to the wear scar. This apparent reorientation of the maximum normal stress may be due to the influence of the higher coefficient of friction in the $x$ sliding direction. It is not known whether the cracking observed at $45^{\circ}$ is actual cleavage or is noncrystallographic in nature.

\section{Conclusions}

The influence of sliding direction on the frictional behavior and surface failure of the plane of natural fluorapatite single crystals was evaluated.

Frictional anisotropy was observed; the coefficient of friction $(\beta)$ in the $x$ direction $(\beta=0.217)$ was approximately $12 \%$ higher than that in the $y$ direction $(\beta=0.193)$. The load at which the ductile-to-brittle transition $(\Omega)$ occurred was significantly higher in the $x$ direction $(\Omega=77 \mathrm{gm})$ than in the $y$ direction $(\Omega=15 \mathrm{gm})$. A mechanism was proposed involving the maximum normal tensile stress and probable cleavage planes.

\section{References}

1. Powers, J.M., and Craig, R.G.: Wear of Fluorapatite Single Crystals: I. A Method for Quantitative Evaluation of Wear, $J$ Dent Res 51: 168-176, 1972.

2. Powers, J.M., and Craig, R.G.: Wear of Fluorapatite Single Crystals: II. Frictional Behavior, I Dent Res 51: 605-610, 1972.

3. Powers, J.M., and Craig, R.G.: Wear of Fluorapatite Single Crystals: III. Classification of Surface Failure, $J$ Dent Res 51:611. $618,1972$.

4. Buckley, D.H.: The Influence of the Atomic Nature of Crystalline Materials on Friction, Am Soc Lubrication Engineers Trans 11: 89. $100,1968$.

5. SteijN, R.P.: On the Wear of Sapphire, $J$ Appl Phys 32: 1951-1958, 1961.

6. Bowden, F.P.; Brookes, C.A.; and Hanwell, A.E.: Anisotropy of Friction in Crystals, Nature (Lond) 203: 27-29, 1964.

7. Bowden, F.P., and Brookes, C.A.: Frictional 
Anisotropy in Non-Metallic Crystals, Proc Roy Soc Lond A295: 244-258, 1966.

8. Time Series Analysis, Ann Arbor, Mich: Statistical Research Laboratory, University of Michigan, 1971.

9. Hertz, H.R.: On the Contact of Elastic Solids, $J$ Reine Angew Math 92: 156-171, 1881 .

10. Katz, J.L., and Ukraincik, K.: On the Anisotropic Elastic Properties of Hydro. xyapatite $J$ Biomechanics 4:221-227, 1971.

11. Hcber, M.T.: Zur Theoric der Beruhrung fester elastischer Korper, Ann Phys (Lpz) 14: 153-163, 1904.

12. TIMoshenko, S.P., and Goodier, J.N.: Theory of Elasticity, 3rd ed, New York: McGrawHill, 1970.

13. Mindlin, R.D.: Compliance of Elastic Bodies in Contact, $J$ Appl Mech 16: 259-268, 1949.
14. Duncan, D.B.: Multiple Range and Multiple F-Test, Biometrics 11:1-42, 1955.

15. DANA, E.S.: A Textbook of Mineralogy, New Iork: John Wiley and Sons, Inc., 1963, p 704.

16. Hopkins, R.H., and KRAMER, W.E.: The Origin of Secondary Phases in Melt-Grown Fluorapatite Crystals, $J$ Electrochem Soc 116: 638-640, 1969.

17. MCManuis, G.M.; Hopkins, R.H.; and TAKeI, W.J.: Dislocation Arrangements in Fluoraapatite, $J$ Appl Phys 40: 180-183, 1969.

18. Hopkins, R.H.: The Origin of Decorated Dislocation Arrays in Fluorapatite, $J$ Crystal Growth 6:91-96, 1969.

19. PowErs, J.M.: The Influence of Crystallographic Direction and Envitonment on the Ductile to Brittle Transition of Fluorapatite Single Crystals under Sliding, dissertation. Inn Arbor, University of Michigan, 1972. 